\title{
The road to rheumatoid arthritis prevention: challenges and opportunities
}

\author{
Yashaar Chaichian $^{1}$ (D) $\cdot$ Mark C. Genovese $^{1}$ (D) $\cdot$ Michael H. Weisman $^{2}$ (iD \\ Received: 18 February 2020 / Revised: 18 February 2020 / Accepted: 28 February 2020 / Published online: 13 March 2020 \\ (C) International League of Associations for Rheumatology (ILAR) 2020
}

\begin{abstract}
Rheumatoid arthritis (RA) is the most prevalent cause of chronic arthritis worldwide and contributes substantial health burden and socioeconomic costs, issues that are magnified by the aging population. Despite significantly improved outcomes in the management of RA with earlier diagnosis and advances in treatment, there is still no cure and disease prevention remains an area of intense interest. Studies examining different treatment regimens in varied subsets of patients with pre-clinical RA have been able to delay but not prevent onset of frank RA. In this timely issue of Clinical Rheumatology, Alpziar-Rodriguez D. and Finckh A. highlight the available literature on pre-clinical RA including modifiable risk factors, results of key intervention trials, and discuss whether prevention of RA is on the horizon. We offer additional thoughts on current areas of uncertainty in RA prevention studies, lessons to be learned from prevention trials in other disease states, and future directions to consider.
\end{abstract}

As the most prevalent autoimmune cause of chronic arthritis worldwide, rheumatoid arthritis (RA) contributes to a significant health burden for affected patients and their families. RA further results in high socioeconomic costs that are compounded by growth of the aging population. An aggressive approach to early diagnosis, adoption of a "treat-to-target" strategy, utilization of combination therapies, and the introduction of targeted biologic and synthetic immunomodulatory agents have all led to significantly improved disease outcomes in RA. However, despite the evolving shift in RA management leading to better disease control in an increasing number of patients, there is still no cure and disease prevention remains elusive. In this timely issue of Clinical Rheumatology, Alpziar-Rodriguez D. and Finckh A. [1] highlight the available literature on pre-clinical RA including modifiable risk factors, results of key intervention trials, and discuss whether prevention of RA is on the horizon.

See related article, https://doi.org/10.1007/s10067-020-04927-6

Yashaar Chaichian

ychaich@stanford.edu

1 Division of Immunology and Rheumatology, Stanford University, Palo Alto, CA, USA

2 Division of Rheumatology, Cedars-Sinai Medical Center, David Geffen School of Medicine, UCLA, Los Angeles, CA, USA

\section{Areas of uncertainty in RA prevention studies and trials}

RA prevention trials have included a wide range of patients considered to be at risk for disease. The heterogeneity of study designs reflects the uncertainty regarding when and in whom to intervene, making it difficult to draw comparisons between studies. Some have focused on first-degree relatives of patients with RA, while others have focused on seropositive patients without joint complaints. Yet other groups have included patients with both immunologic features and musculoskeletal symptoms and signs that were deemed either short of overt disease or that did not reach the threshold of disease classification criteria. The authors correctly point out that seropositivity has been inconsistently defined in pre-clinical RA trials thus far. Whether to include both RF and ACPA-positive patients versus those who have just one positive autoantibody, and what titer cut-off and assay should be used, needs consensus before proceeding with additional studies.

The all-important question of when exactly within the spectrum of pre-clinical RA patients transition from being "at-risk" to "destined" to develop RA is unknown. Several prediction tools have been devised to help stratify patients into low, intermediate, and high-risk of subsequent RA. However, these tools require additional validation and refinement. Furthermore, a consensus prediction tool has yet to be developed. Knowing exactly when the odds of early intervention are in favor of prevention is of course highly relevant for 
several reasons. First, it would provide valuable prognostic information to the patient, family, and treating physician. Secondly and perhaps more importantly, it would facilitate pre-clinical RA trials that directly target the population for which intervention could potentially prevent disease.

Until we have better prediction models, we are left wondering which exact patients to target in RA prevention trials. For example, should seropositive patients with arthralgias (but not frank arthritis) be included? How different are these patients from those without symptoms or yet those meeting the threshold of disease classification criteria? Is early treatment in pre-clinical RA more akin to secondary, rather than primary, prevention? One may reasonably assume that the inflammatory disease cascade may have reached a tipping point and be too aberrant to prevent onset of chronic disease. Perhaps focusing on seropositive RA patients without any joint complaints, i.e. those who may be "earlier" within the disease trajectory, may be a more apt group to target in intervention trials. While intervening at this early stage may be more likely to prevent the development of subsequent RA, it is also reasonable to assume that many of these patients will never progress to chronic recognizable RA. Finally, it is assumed that any prevention strategy must contain an intervention that is safe enough to avoid harm, particularly in those not destined to develop the disease. How many cases of RA could one prevent with early intervention that would be worth the potential risks associated with immunosuppression?

\section{Lessons from prevention trials outside of RA}

Primary (and secondary) prevention trials in other disease states are instructive regarding the question of benefit versus harm as it relates to pharmacotherapy. In terms of primary prevention, an example is the study of aspirin in patients without a history of cardiovascular disease to determine if this intervention could prevent this outcome. A meta-analysis [2] of the relevant studies showed that aspirin did lower the risk for the combined endpoint of non-fatal myocardial infarction (MI) and fatal coronary heart disease. However, this came at the expense of an elevated risk for hemorrhagic strokes and major gastrointestinal bleeding. Regarding secondary prevention, the CANTOS study [3], designed as a proof of principle regarding the contribution of inflammation causing atherosclerotic cardiovascular disease, is an excellent example of this dilemma. The study included high risk patients with a prior $\mathrm{MI}$ and elevated high-sensitivity $\mathrm{C}$-reactive protein who were randomized to three different doses of canakinumab versus placebo, with the primary outcome of interest being additional evidence for non-fatal MI, non-fatal stroke, or cardiovascular death. While canakinumab at a dose of $150 \mathrm{mg}$ every 3 months significantly lowered the rate of recurrent cardiovascular events (independent of lipid-lowering), it also led to a higher incidence of fatal infection compared to placebo as a significant trade-off. Certainly, the patient population and outcomes studied in the above two examples - especially regarding the risks of the intervention as well as the outcome to be prevented - are quite different compared to RA prevention trials. However, the results illustrate the potential and reallife implications of intervening - both positive and negative for which the research community needs to be mindful of when designing and implementing prevention trials in other disease states, including RA.

\section{Deferred Hope in preventing RA}

Beyond the question of acceptable risk, as Alpziar-Rodriguez D. and Finckh A. note, the results of RA prevention trials thus far have been relatively disappointing. Several RA prevention studies did demonstrate the ability of pharmacotherapy to delay onset of disease, including: the PROMPT study [4] among the ACPA-positive subset of patients treated with low-dose methotrexate for early undifferentiated arthritis, the ADJUST trial [5] using abatacept in ACPA-positive patients with undifferentiated arthritis plus symptomatic clinical synovitis of at least 2 joints, and the PRAIRI study [6] using rituximab in double-positive (IgM-RF and ACPA) patients with arthralgias plus at least one of the following: $\mathrm{CRP}>0.6 \mathrm{mg} / \mathrm{L}$ at screening or subclinical synovitis on ultrasound or MRI. Nevertheless, the fact remains that no pre-clinical RA trial to date has been able to prevent the development of RA in a population of patients. This conclusion is true not only among those studies that focused on patients considered "later" in the disease pathway (e.g. seropositive plus arthralgias), but also studies that included patients who were seropositive but had yet to manifest joint complaints.

These results do not necessarily suggest that early intervention in future trials will fail to prevent RA. It is possible that the lack of success among existing RA prevention trials is because the exact subgroup of patients, timepoint to intervene, specific intervention, follow-up duration, or some combination thereof has yet to be identified. The authors correctly point out that the number of studies including subjects with early pre-clinical disease who were treated with pharmacotherapy is relatively small, and several studies are underway that may help answer this question. Further data on the impact of RA prevention through lifestyle modification among at-risk populations (e.g. smokers, those with poor gingival health, industrial/environmental exposure [7]) is also needed, to determine if a non-pharmacologic approach may succeed. While these types of studies would have low/no risk of harm, they undoubtedly require many more patients and years of followup compared to pharmacotherapeutic intervention trials in RA. 


\section{Conclusions and future directions}

We close with several thoughts. First, the RA research community should collaboratively and uniformly classify the subset of patients with pre-clinical RA to be included in future prevention trials. It will help ensure that any intervention is applied only to the subset of patients at a stage within the preclinical pathway still likely to be reversible, and for whom intervention carries acceptable (rather than undue) risk. Identifying this exact subset of patients and timepoint within the trajectory toward RA has thus far been elusive. The theoretical biomarker, or even the genetic/epigenetic readout, has not been identified to guide that patient selection process. Lessons learned from prevention studies in disease states outside of RA can be instructive in terms of the question of acceptable risk. We also believe that input from patients and other relevant stakeholders should be considered to ensure that future decisions on RA prevention trials take everyone's interests into account. Since RA management today has reached a point where the most dreaded complications of the disease are rarely seen, patients may have a different view of disease prevention risks and benefits. Lastly, the total cost of RA prevention trials, including the challenges involved in recruitment and retention of study subjects, as well as study duration, needs to be discussed in greater detail. Beyond the direct monetary costs related to study design and implementation, there are costs associated with the intervention and management of potential side effects and complications of therapy. In addition, there are unmeasurable costs related to the impact on patients' quality of life, as well as potential future costs related to loss of health insurance coverage for some patients classified as having a possible autoimmune disease. Ultimately, there are no easy answers, but these are issues worth a much broader discussion.

\section{Compliance with ethical standards}

Conflicts of interest YC has received support from Gilead Sciences, AMPEL BioSolutions, Pfizer, and GSK. MCG has received grants/ contracts from Abbvie, Gilead, GSK, Regeneron, and RPharm, and consulted for Amgen, Abbvie, Lilly, Genentech/Roche, Gilead, GSK, Sanofi, Regeneron, and RPharm. MHW has no disclosures.

\section{References}

1. Alpizar-Rodriguez D, Finckh A (2020) Is the prevention of rheumatoid arthritis possible? Clin Rheumatol:1-7. https://doi.org/10.1007/ s10067-020-04927-6

2. Hayden M, Pignone M, Phillips C, Mulrow C (2002) Aspirin for the primary prevention of cardiovascular events: a summary of the evidence for the U.S. preventive services task force. Ann Intern Med 136:161-172

3. Ridker PM, Everett BM, Thuren T, MacFadyen J, Chang WH, Ballantyne C, Fonseca F, Nicolau J, Koenig W, Anker SD, Kastelein JJP, Cornel JH, Pais P, Pella D, Genest J, Cifkova R, Lorenzatti A, Forster T, Kobalava Z, Vida-Simiti L, Flather M, Shimokawa H, Ogawa H, Dellborg M, Rossi PRF, Troquay RPT, Libby P, Glynn RJ, CANTOS Trial Group. (2017) Antiinflammatory therapy with canakinumab for atherosclerotic disease. N Engl J Med 377:1119-1131

4. van Dongen H, van Aken J, Lard LR, Visser K, Ronday HK, Hulsmans HM, Speyer I, Westedt ML, Peeters AJ, Allaart CF, Toes RE, Breedveld FC, Huizinga TW (2007) Efficacy of methotrexate treatment in patients with probable rheumatoid arthritis: a double-blind, randomized, placebo-controlled trial. Arthritis Rheum 56:1424-1432

5. Emery P, Durez P, Dougados M, Legerton CW, Becker JC, Vratsanos G, Genant HK, Peterfy C, Mitra P, Overfield S, Qi K, Westhovens R (2010) Impact of T-cell costimulation modulation in patients with undifferentiated inflammatory arthritis or very early rheumatoid arthritis: a clinical and imaging study of abatacept (the ADJUST trial). Ann Rheum Dis 69:510-516

6. Gerlag DM, Safy M, Maijer KI, Tang MW, Tas SW, Starmans-Kool MJF, van Tubergen A, Janssen M, de Hair M, Hansson M, de Vries N, Zwinderman AH, Tak PP (2019) Effects of B-cell directed therapy on the preclinical stage of rheumatoid arthritis: the PRAIRI study. Ann Rheum Dis 78:179-185

7. Zhao N, Smargiassi A, Hatzopoulou M et al (2019) Multiple industrial air pollutants and anti-citrullinated protein antibody positivity [abstract]. Arthritis Rheumatol 71(suppl 10)

Publisher's note Springer Nature remains neutral with regard to jurisdictional claims in published maps and institutional affiliations. 